\title{
Práticas alimentares no primeiro ano de vida
}

\author{
Eating habits in the first year of life \\ Hábitos alimenticios en el primer año de vida
}

\section{Ana Paula Esmeraldo Lima', Marly Javorski", Maria Gorete Lucena de Vasconcelos" \\ ' Universidade de Pernambuco, Faculdade de Enfermagem Nossa Senhora das Graças. Prefeitura Municipal do Recife. Recife-PE, Brasil. \\ "Universidade Federal de Pernambuco, Departamento de Enfermagem. Recife-PE, Brasil.}

Submissão: 11-09-2010 Aprovação: 08-12-2011

\begin{abstract}
RESUMO
O estudo buscou analisar a literatura publicada referente às práticas alimentares no primeiro ano de vida de filhos de mães adolescentes, por meio de uma revisão integrativa nas bases de dados Lilacs, Medline e Cochrane, no período de 2000 a 2010. Foram utilizadas as palavras-chave: adolescente, aleitamento materno, alimentação mista e nutrição do lactente, sendo selecionados onze artigos científicos, que compuseram a amostra do estudo. Os resultados apontaram a incipiência de pesquisas publicadas sobre o tema proposto, sobretudo para as que versam sobre alimentação complementar, desvelando uma lacuna na produção mundial sobre o tema. Novas pesquisas, portanto, precisam ser desenvolvidas com foco em mães adolescentes, devendo-se explorar a alimentação infantil em toda sua dimensão.
\end{abstract}

Descritores: Adolescente; Aleitamento materno; Alimentação mista; Nutrição do lactente.

\begin{abstract}
The study aimed to examine the relevant published literature to food practices in the first year of life of teenage mothers' children, through an integrative review within Lilacs, Medline and Cochrane, databases from 2000 to 2010. We used the keywords: adolescent, breastfeeding, mixed feeding and infant nutrition, from which we selected eleven articles that composed the study sample. The results indicated the paucity of published research on the proposed topic, especially for those focusing on complementary feeding, revealing a gap in global production on the subject. New researches, therefore, need to be developed with a focus on teenage mothers, exploring infant feeding in all its dimensions.
\end{abstract}

Key words: Adolescent; Breastfeeding; Mixed feeding; Infant nutrition.

\section{RESUMEN}

El estudio trató de examinar la bibliografía publicada sobre los hábitos alimenticios en el primer año de vida de los hijos de madres adolescentes, mediante una revisión integradora en las bases de datos Lilacs, Medline y Cochrane, en el período de 2000 a 2010. Hemos usado las palabras clave: adolescente, lactancia materna, alimentación mixta y nutrición del lactante, siendo seleccionados once trabajos, los cuales compusieran la muestra del estudio. Los resultados indicaron la escasez de investigaciones publicadas sobre el tema propuesto, especialmente para aquellos que se ocupan de la alimentación complementaria, con una brecha en la producción mundial sobre el tema. Nuevos estudios, por lo tanto, necesitan ser desarrollados con un enfoque en las madres adolescentes, y explorar la alimentación infantil en todas sus dimensiones.

Palabras clave: Adolescente; Lactancia materna; Alimentación mixta; Nutrición del lactante. 


\section{INTRODUÇÃO}

A adolescência é considerada como o processo de passagem da vida infantil para a vida adulta e tem sua conceituação influenciada por processos históricos, com diferentes significados em diversas classes sociais, épocas e culturas ${ }^{(1)}$.

Para a Organização Mundial de Saúde (OMS), a adolescência está circunscrita à segunda década de vida, entre os $10 \mathrm{e}$ 19 anos de idade, podendo, ainda, ser subdividida em adolescência menor (de 10 a 14 anos) e adolescência maior (de 15 a 19 anos). Tal fase deve ser entendida como um fenômeno singular, caracterizado por influências socioculturais que vão se concretizando por meio de reformulações constantes de caráter social, sexual e de gênero ${ }^{(2)}$.

A sexualidade, embora presente desde o nascimento, é consolidada na adolescência, quando se estabelecem as condições para as funções sexuais do adulto. O que se observa nos últimos anos, entretanto, é que, cada vez mais cedo, grande número de jovens assume vida sexual ativa, e é nesse contexto que a gravidez na adolescência constitui-se um grave problema de saúde pública(1).

A insuficiente orientação do jovem sobre seu corpo, sua sexualidade e seus direitos reprodutivos contribui para a falta de controle da fecundidade, afetando amplamente as mulheres brasileiras, especialmente as de condições socioeconômicas menos favorecidas. A gestação precoce traz problemas de natureza biológica e social, não só para a mulher, mas também para o concepto. A falta de perspectivas de futuro, entretanto, faz com que algumas adolescentes representem a gravidez como um meio para o alcance do status de mulher adulta e até, para o resgate de sua cidadania ${ }^{(3)}$.

A ocorrência de gravidez e maternidade nessa fase da vida traz como conseqüência mudanças que exigem uma redefinição no futuro com um filho, geralmente, não planejado ${ }^{(4)}$. A adolescente vivencia intensa reestruturação e amadurecimento pessoal e social, acarretando mudança de identidade e nova definição de papéis. A prática de cuidar do filho exige, portanto, maior esforço de adaptação, que deve ser gradativa, na medida em que vai alterando sua condição de filha adolescente para mãe adolescente ${ }^{(5)}$.

Como qualquer mulher que vivencia a maternagem, a jovem mãe irá desenvolver um papel fundamental em relação ao cuidado com o filho, sendo o cuidado com a alimentação um dos mais importantes, haja vista as repercussões que determina na saúde do infante.

A formação dos hábitos alimentares, então, tem como influência de maior destaque a interação da criança com a própria mãe ou com a pessoa mais ligada a sua alimentação, além do ambiente doméstico, das condições socioeconômicas e das relações familiares. Desse modo, a família poderá determinar a adoção de práticas de alimentação, favorecendo o estabelecimento de um padrão de comportamento alimentar infantil adequado ou não(6).

A alimentação dos adolescentes, por sua vez, pouco frequentemente pode ser considerada adequada. Mesmo quando os hábitos alimentares da família são elogiáveis, o mesmo não se pode afirmar do filho adolescente, que tende a rejeitar os hábitos alimentares da família e a adotar os hábitos do seu grupo de convivência. As peculiaridades características desta fase, assim, podem contribuir negativamente na instituição da alimentação do filho. Mães com hábitos alimentares inadequados dificilmente irão estabelecer uma alimentação infantil adequada.

O aleitamento materno exclusivo durante os seis primeiros meses de vida, a alimentação complementar oportuna e adequada e a manutenção do aleitamento materno complementado até os dois anos de idade ou mais é o esquema recomendado pela Organização Mundial de Saúde (OMS) para as crianças de todo o mundo. Entre os fatores de risco para a baixa taxa de aleitamento materno exclusivo e continuado e introdução precoce de alimentos sólidos, a maternidade na adolescência se destaca como um dos principais. Recentemente, a OMS lançou documento alertando acerca dos riscos de adoecer e morrer para os filhos de mães adolescentes, enfatizando a necessidade dos profissionais da saúde estarem capacitados a trabalhar com este grupo, sobretudo no apoio à alimentação infantil adequada ${ }^{(7-9)}$.

Neste cenário, evidenciou-se a necessidade de buscar na literatura referências sobre as práticas de alimentação de filhos de mães adolescentes, a fim de responder ao questionamento: como são instituídas as práticas de alimentação no primeiro ano de vida de filhos de mães adolescentes? A opção pelo desenvolvimento de uma revisão integrativa sobre esse tema deve-se ao fato de que tal revisão possibilita a interpretação de estudos produzidos sobre práticas de alimentação infantil, abrangendo desde o aleitamento materno até a alimentação complementar, que tenham como foco as mães adolescentes, no intuito de compreender como é estabelecida a alimentação dessas crianças, auxiliando no desenvolvimento de futuras investigações.

O presente estudo tem como objetivo, pois, analisar a literatura publicada referente às práticas alimentares no primeiro ano de vida de filhos de mães adolescentes.

\section{METODOLOGIA}

A fim de alcançar o objetivo proposto, optou-se pelo método da revisão integrativa, visto que ele possibilita reunir e sintetizar resultados de pesquisas sobre um determinado tema ou questão, de maneira sistemática e ordenada, contribuindo para o aprofundamento do tema investigado, além de apontar lacunas do conhecimento que precisam ser preenchidas com a realização de novas pesquisas. A realização de uma revisão integrativa exige os mesmos padrões de rigor e clareza utilizada nos estudos primários ${ }^{(10-11)}$.

Para a construção dessa revisão, os seguintes passos foram percorridos: elaboração da questão norteadora, estabelecimento de critérios para seleção da amostra, elaboração de um instrumento para coleta de dados, análise crítica dos dados, interpretação e apresentação dos resultados ${ }^{(12)}$.

O levantamento bibliográfico foi realizado no período de fevereiro a abril de 2010, nas seguintes bases de dados: Literatura Latino-Americana e do Caribe em Ciências da Saúde (LILACS), Medical Literature Analysis and Retrieval Sistem 
Tabela 1. Artigos encontrados e selecionados, segundo bases de dados pesquisadas.

\begin{tabular}{|c|c|c|c|c|c|c|}
\hline \multirow{2}{*}{$\begin{array}{l}\text { Base de dados } \\
\text { Descritores }\end{array}$} & \multicolumn{2}{|c|}{ LILACS } & \multicolumn{2}{|c|}{ MEDLINE } & \multicolumn{2}{|c|}{ COCHRANE } \\
\hline & $\begin{array}{c}\text { Artigos } \\
\text { encontrados }\end{array}$ & $\begin{array}{c}\text { Artigos } \\
\text { selecionados }\end{array}$ & $\begin{array}{c}\text { Artigos } \\
\text { encontrados }\end{array}$ & $\begin{array}{c}\text { Artigos } \\
\text { selecionados }\end{array}$ & $\begin{array}{c}\text { Artigos } \\
\text { encontrados }\end{array}$ & $\begin{array}{c}\text { Artigos } \\
\text { selecionados }\end{array}$ \\
\hline Adolescente, nutrição do lactente & 160 & 01 & 00 & - & 11 & 01 \\
\hline Adolescente, aleitamento materno & 166 & 07 & 775 & 13 & 406 & - \\
\hline Adolescente, alimentação mista & 04 & - & 00 & - & 153 & - \\
\hline Total & 330 & 08 & 775 & 13 & 570 & 01 \\
\hline
\end{tabular}

on-line (MEDLINE) e Biblioteca Cochrane (COCHRANE).

Foram utilizados, para a busca dos artigos, os seguintes descritores e suas combinações nas línguas inglesa e espanhola: Adolescente, Aleitamento materno, Alimentação mista e Nutrição do lactente. O cruzamento dos referidos descritores foi realizado da seguinte forma: adolescente e aleitamento materno, adolescente e alimentação mista, e adolescente e nutrição do lactente. A tabela 1 descreve o caminho percorrido no levantamento dos artigos.

Os critérios utilizados para a seleção da amostra foram: artigos publicados em português, inglês ou espanhol; artigos publicados nos referidos bancos de dados no período de 2000 a 2010, que retratassem a temática das práticas de alimentação infantil de filhos de mães adolescentes, abrangendo experiências e atitudes frente ao aleitamento materno e/ou à alimentação complementar.

Uma vez acessados os títulos e resumos das publicações, 22 artigos foram selecionados e adquiridos na íntegra. Três artigos não estavam disponíveis como texto completo e foram solicitados diretamente aos autores, que prontamente disponibilizaram suas publicações. De posse de todas as cópias, procedeu-se à leitura integral e crítica dos 22 artigos quanto aos critérios de inclusão, ficando a amostra final composta por onze artigos científicos.

Para facilitar a coleta de dados, foi desenvolvido um instrumento, preenchido para cada artigo da amostra final do estudo, contendo itens como título e autores, local de publicação, tipo de estudo, objetivos, resultados, implicações, limitações, dentre outros. A utilização de tal instrumento permitiu a sucinta organização dos dados, facilitando a comparação dos estudos em tópicos específicos. Os resultados extraídos de cada estudo foram analisados de forma descritiva, discutidos à luz da literatura específica.

\section{RESULTADOS E DISCUSSÃO}

A amostra final desta revisão integrativa foi composta por onze artigos científicos, selecionados pelos critérios previamente descritos. Desses, a maioria foi identificada na base de dados MEDLINE, seguida pela base de dados LILACS e, por fim, COCHRANE. A caracterização de cada um desses artigos encontra-se descrita no Quadro 1.

Pode-se observar a incipiência de artigos científicos publicados sobre o tema proposto. Apesar do elevado quantitativo de estudos sobre as práticas de alimentação infantil, ainda são escassos aqueles que focam os filhos de mães adolescentes, grupo considerado de risco para instituição de práticas de alimentação inadequadas.

Importante salientar, ainda, que entre os poucos artigos encontrados, quase a totalidade versa sobre aleitamento materno. As palavras-chave mais utilizadas pelos autores foram "adolescente" e "aleitamento materno", presentes em 10 $(90,9 \%)$ estudos. Percebe-se, assim, que os descritores "nutrição do lactente" (encontrado em apenas um artigo) e "alimentação mista" (ausente nos estudos selecionados), apesar de serem os que melhor representam a alimentação no primeiro ano de vida, são pouco utilizados pelos pesquisadores. A nítida carência de artigos sobre alimentação complementar sugere a subestima da sua importância para a saúde da criança.

Ao analisar o país de origem dos estudos selecionados, percebe-se a predominância dos Estados Unidos, seguido do Brasil. Juntos, esses países respondem por mais de $80 \%$ das publicações. A ausência de publicações em língua espanhola evidencia a necessidade de pesquisas sobre práticas de alimentação infantil nestes países, já que o tema adquire peculiaridades conforme o contexto sócio-cultural em que está inserido. Esta necessidade é enfatizada por estudo norte-americano ${ }^{(13)}$, que buscou examinar decisões e práticas de aleitamento materno entre adolescentes latino-americanas e afro-americanas, reconhecendo a importância do valor cultural para as práticas de alimentação.

Quanto à periodicidade das publicações, observa-se uma distribuição quase que uniforme de produção anual, já que, proporcionalmente, foi publicado aproximadamente um artigo por ano. No entanto, é possível notar que $36 \%$ das publicações concentraram-se nos últimos dois anos, o que pode sinalizar para uma tendência à maior ênfase nas práticas de alimentação de filhos de mães adolescentes. Dos periódicos identificados, apenas dois publicaram mais de um artigo sobre o tema, sendo um referente à Enfermagem Materno-Infantil e o outro destinado a estudos sobre aleitamento materno.

Verifica-se que todos os artigos apresentam os objetivos da pesquisa de forma clara, facilitando o entendimento do leitor. Apenas um estudo não expôs com clareza o problema de pesquisa; cinco $(45,4 \%)$ dos artigos evidenciaram como problema de pesquisa a necessidade de estudos sobre alimentação infantil na perspectiva de mães adolescentes, reconhecendo a vulnerabilidade desse grupo.

Em relação à abordagem metodológica empregada, identifica-se que cinco $(45,4 \%)$ utilizaram metodologia quantitativa, 


\begin{tabular}{|c|c|c|c|}
\hline Base de dados & Título & Objetivo & País \\
\hline MEDLINE & $\begin{array}{l}\text { Adolescent attitudes, beliefs and concerns } \\
\text { regarding breastfeeding }\end{array}$ & $\begin{array}{l}\text { Investigar as atitudes, crenças e preocupações } \\
\text { de adolescentes grávidas e puérperas quanto ao } \\
\text { aleitamento materno }\end{array}$ & Estados Unidos \\
\hline MEDLINE & $\begin{array}{l}\text { Breastfeeding experiences of urban adolescent } \\
\text { mothers }^{(17)}\end{array}$ & $\begin{array}{l}\text { Examinar a experiência de mães adolescentes em } \\
\text { amamentação }\end{array}$ & Estados Unidos \\
\hline MEDLINE & $\begin{array}{l}\text { The influence of adolescent mothers' breastfeeding } \\
\text { confidence and attitudes on breastfeeding initiation } \\
\text { and duration }\end{array}$ & $\begin{array}{l}\text { Analisar a influência da confiança e atitudes } \\
\text { de mães adolescentes no início e duração do } \\
\text { aleitamento materno }\end{array}$ & Canadá \\
\hline LILACS & $\begin{array}{l}\text { Fatores relacionados às dificuldades no } \\
\text { aleitamento materno entre mães adolescentes da } \\
\text { Fundação Santa Casa de Misericórdia do Pará(23) }\end{array}$ & $\begin{array}{l}\text { Verificar os fatores que dificultam a amamentação } \\
\text { entre mães adolescentes }\end{array}$ & Brasil \\
\hline LILACS & $\begin{array}{l}\text { Promoção do aleitamento materno com mães } \\
\text { adolescentes: acompanhando e avaliando essa } \\
\text { prática }^{(24)}\end{array}$ & $\begin{array}{l}\text { Avaliar a qualidade do aleitamento materno entre } \\
\text { mães adolescentes }\end{array}$ & Brasil \\
\hline MEDLINE & Breastfeeding behaviors and experiences of ${ }^{(18)}$ & $\begin{array}{l}\text { Analisar as experiências e comportamentos } \\
\text { relacionados com amamentação de mães } \\
\text { adolescentes }\end{array}$ & Estados Unidos \\
\hline LILACS & $\begin{array}{l}\text { Amamentação entre mães adolescentes e não } \\
\text { adolescentes, Monte Carlos, } \mathbf{M G}^{(27)}\end{array}$ & $\begin{array}{l}\text { Estimar a prevalência de amamentação entre } \\
\text { mães adolescentes e não-adolescentes e os fatores } \\
\text { associados ao desmame }\end{array}$ & Brasil \\
\hline MEDLINE & $\begin{array}{l}\text { Adolescent mothers and breastfeeding: experiences } \\
\text { and support needs - na exploratory study }{ }^{(19)}\end{array}$ & $\begin{array}{l}\text { Explorar as vivências e necessidades de apoio de } \\
\text { mães adolescentes que iniciam a amamentação }\end{array}$ & Inglaterra \\
\hline $\begin{array}{l}\text { LILACS/ } \\
\text { MEDLINE }\end{array}$ & $\begin{array}{l}\text { A amamentação e a alimentação complementar de } \\
\text { filhos de mães adolescentes são diferentes das de } \\
\text { filhos de mães adultas? }{ }^{(4)}\end{array}$ & $\begin{array}{l}\text { Comparar o aleitamento materno e a alimentação } \\
\text { complementar de filhos de mães adolescentes e } \\
\text { não-adolescentes }\end{array}$ & Brasil \\
\hline COCHRANE & $\begin{array}{l}\text { Home and videotape intervention delays early } \\
\text { complementary feeding among adolescent } \\
\text { mothers }\end{array}$ & $\begin{array}{l}\text { Avaliar a eficácia de uma intervenção para } \\
\text { retardar a introdução precoce da alimentação } \\
\text { complementar entre mães adolescentes }\end{array}$ & Estados Unidos \\
\hline MEDLINE & $\begin{array}{l}\text { African-American and Latina adolescent mothers' } \\
\text { infant feeding decisions and breastfeeding } \\
\text { practices: a qualitative study }{ }^{(13)}\end{array}$ & $\begin{array}{l}\text { Examinar, a partir da perspectiva da adolescente, } \\
\text { a tomada de decisão da alimentação infantil no } \\
\text { período pré e pós-natal }\end{array}$ & Estados Unidos \\
\hline
\end{tabular}

Quadro 1. Caracterização dos artigos selecionados

cinco $(45,4 \%)$ realizaram uma abordagem qualitativa e uma desenvolveu pesquisa quanti-qualitativa (descritiva). As abordagens quantitativa e qualitativa se apóiam, fornecendo um quadro mais geral do conhecimento das práticas alimentares. São métodos complementares, e não competitivos, devendo sua escolha ser baseada na natureza do problema de pesquisa $^{(14)}$.

Entre os estudos quantitativos, três são não-experimentais, abrangendo estudo transversal e de coorte. Um utiliza o desenho quase-experimental, através de estudo correlacional e outro adota o desenho experimental, com um estudo caso-controle.

Desperta a atenção o fato de que nenhum dos estudos qualitativos explicitou a utilização de algum referencial teórico para embasar a análise e interpretação do fenômeno estudado. Apesar de um destes artigos referir a realização de uma "entrevista etnográfica", não há qualquer menção a utilização da etnografia para análise dos dados.

A maioria dos estudos (90,9\%) optou por uma seleção amostral por conveniência, contabilizando um total de 883 adolescentes ao se somar as amostras dos 11 artigos. No entanto, apenas três $(27,3 \%)$ justificam o tamanho amostral, ficando aos demais a incerteza da representatividade da população que se pretende investigar.

A caracterização dos sujeitos, embora não esteja descrita em alguns estudos, apresentou aparente homogeneidade, onde a média de idade das adolescentes foi de 16,5 anos; a maioria apresentava baixa escolaridade, embora algumas ainda estivessem cursando o ensino médio; a renda familiar era baixa, sendo que poucas adolescentes contribuíam com essa renda; a maior parte era primípara e estava vivenciando pela primeira vez, portanto, a experiência de alimentar o filho.

No que se refere à temática dos artigos, como já citado anteriormente, há predominância do tema aleitamento materno, sendo exploradas, principalmente, as experiências maternas $(45,4 \%)$. Ainda, dois $(18,2 \%)$ dos artigos focalizaram as dificuldades na amamentação, dois compararam a prática do aleitamento materno em adolescentes e não adolescentes, um analisou a influência da confiança e atitudes de gestantes na instituição do aleitamento materno e, por fim, outro avalia 
a eficácia de uma intervenção para retardar o início da alimentação complementar. O tema alimentação complementar, presente em dois estudos, mostrou-se como um "coadjuvante", restrito à identificação dos alimentos consumidos na ocasião das entrevistas.

O Ministério da Saúde reconhece a ênfase à promoção do aleitamento materno nas últimas décadas, ao mesmo tempo em que uma lacuna de informação se abria quanto à alimentação complementar ${ }^{(15)}$. A alimentação da criança no primeiro ano de vida, por sua vez, se constitui elemento fundamental para garantir sua sobrevivência e crescimento adequado. Deste modo, além de receber o leite materno, a criança necessita de uma alimentação adequada e oportuna a partir dos seis meses de vida.

A alimentação infantil, por sua vez, deve ser estudada em sua totalidade, o que significa investigar não somente o registro de freqüência e quantidade dos alimentos consumidos, mas os fatores decisórios para escolha do alimento, as formas de preparar e oferecê-lo, contemplando os aspectos socioculturais envolvidos no fenômeno da alimentação(16).

Dos cinco artigos que analisaram as experiências maternas em aleitamento materno, nenhum foi produzido no Brasil. Nesses, as adolescentes atribuíram à decisão de amamentar os benefícios do aleitamento materno, principalmente para a saúde da criança. Referiram, também, que esta decisão pertencia a elas, independente da opinião dos outros, embora algumas tenham admitido a influência de mães, profissionais, familiares, amigos e professores ${ }^{(13,17-20)}$. Naquelas pesquisas em que se trabalhou com adolescentes no pré e pós-natal, é possível observar a baixa taxa de início do aleitamento materno ${ }^{(13,21-22)}$. Tal fato pode ser justificado pela ausência do título Hospital Amigo da Criança nas instituições sede das pesquisas, já que tal título caracteriza o hospital como incentivador da amamentação.

Vários fatores também foram apontados como problemas para manutenção do aleitamento materno, entre eles dor nas mamas, cansaço, frustração, leite insuficiente, ou fraco, retorno à escola, limitação das atividades cotidianas, ou medo da criança recusar mamadeira e ficar muito dependente da mãe. Importante enfatizar que o constrangimento de amamentar em público foi um dos principais fatores referidos pelas adolescentes, relato este pouco comum no nosso país, já que esta percepção negativa de amamentar em público depende da cultura de cada grupo social. Em estudo realizado em Chicago, EUA, com adolescentes de diferentes etnias, várias adolescentes latinas referiram que esta cultura norte-americana de considerar o aleitamento materno em público constrangedor e vergonhoso não existe em seus países de origem ${ }^{(13)}$.

Ambos os artigos que focalizaram nas dificuldades em amamentar são nacionais e identificaram a dor, as dificuldades na pega e a posição inadequada como os principais problemas para a instituição da amamentação. A oferta de água e chá, principalmente por influência das avós, também surgem como importante fator para interrupção do aleitamento materno. O maior número de filhos, em um desses estudos, mostrou-se determinante para diminuir as dificuldades na amamentação ${ }^{(23-24)}$.
A cultura institucional frequentemente atribui à amamentação um processo natural e instintivo e pode contribuir para o surgimento de sentimentos conflituosos para a mãe que se depara com estas dificuldades. Os profissionais da saúde exercem, então, papel fundamental na promoção do aleitamento materno, na medida em que são capazes de reconhecer as vicissitudes do amamentar e apoiar a nutriz em seu processo decisório(25).

A necessidade de uma rede de apoio para as jovens mães é salientada em uma das pesquisas, na qual se inclui o apoio emocional, encorajamento, apoio técnico/assistencial, orientações e apoio da rede social, onde se incluem família, amigos, pares, profissionais ${ }^{(19)}$. Para problemas como retorno à escola ou constrangimento de amamentar em público, o uso de bombas de ordenha é frequentemente estimulado ${ }^{(13,17)}$. Esta recomendação, por sua vez, ainda é pouco comum no Brasil, tendo em vista o elevado custo de tal equipamento, sendo orientada, então, a ordenha manual.

Para estudo realizado em Manitoba, Canadá, as adolescentes que apresentavam maior confiança, principalmente no período pós-natal, tinham mais probabilidade de amamentar seus filhos, e recomenda que os profissionais sejam encorajados a promover a confiança no aleitamento materno, desde o pré-natal, dissipando mitos e oferecendo suporte necessário(22).

Em uma pesquisa de intervenção, incluída na amostra, verificou-se que através de visitas domiciliares e exibição de vídeo com adolescentes nutrizes, as mães mostram-se quatro vezes mais propensas a aderir às recomendações de introdução da alimentação complementar a partir dos 4-6 meses de vida. O grupo de intervenção também se mostrou mais capaz de desafiar o preconceito cultural, e negociar com suas próprias mães o manejo da alimentação dos seus filhos ${ }^{(21)}$.

As avós podem influenciar negativamente no aleitamento materno, seja na duração ou no seu caráter exclusivo, o que pode ser justificado pelas experiências destas na alimentação dos seus filhos, já que há algumas décadas o aleitamento artificial era amplamente divulgado e praticado, juntamente com a oferta de água, chás e sucos, ao mesmo tempo em que se acreditava que o leite materno era fraco e insuficiente para nutrir uma criança ${ }^{(26)}$. A influência de fatores sociais e culturais, portanto, não deve ser desconsiderada ao se elaborar ações para promoção do aleitamento materno e introdução da alimentação complementar adequada e em momento oportuno. Neste contexto, a visita domiciliar torna-se fundamental para orientar o papel da família e incentivar e apoiar a nutriz.

Entre os estudos que compararam as práticas de adolescentes e não adolescentes, não se evidenciaram diferença estatística significativas de aleitamento materno entre os grupos. A ocorrência de dificuldades para amamentar, no entanto, foi mais freqüente em mães adolescentes, mostrando-se fortemente associada ao desmame ${ }^{(27)}$. Os filhos de mães adolescentes, ainda, apresentaram estatura ao nascer e peso e IMC com um ano de vida menores que os de adultas, além de ingerir significativamente menos carne ${ }^{(4)}$.

Segundo a Organização Mundial de Saúde, as taxas de baixo peso ao nascer também são mais elevadas entre os fiIhos de adolescentes, e quanto mais jovem a mãe maior o risco de morte durante o primeiro mês de vida ${ }^{(9)}$. Quanto à 
amamentação, estudo realizado em Campinas traz resultados discordantes, onde mães adolescentes ofereceram leite materno por menos tempo aos seus filhos ${ }^{(28)}$. Nos Estados Unidos, bem como em outros países desenvolvidos, as mães adolescentes continuam a ter as menores taxas de aleitamento materno ${ }^{(8)}$. Além do risco mais elevado de desmamar seus filhos precocemente, as jovens mães também são mais propensas a erros na introdução alimentar, seja pelo seu baixo poder aquisitivo ou pela simples repetição do seu hábito alimentar, geralmente inadequado.

As adolescentes são mais propensas a oferecerem aos seus filhos alimentos comumente consumidos por elas, como refrigerantes, bolachas e salgadinhos, que são, por sua vez, inadequados para crianças menores de um ano ${ }^{(29)}$. É na infância, no entanto, que o hábito alimentar é estabelecido, quando as crianças começam a receber a alimentação complementar. Nessa fase, as atitudes em relação à comida são normalmente aprendidas, transmitidas por pessoas cuja relação afetiva é grande, comumente a mãe $\mathrm{e}^{(30)}$.

Compreender a experiência de alimentar o filho para a mãe adolescente implica em uma atuação mais eficaz do profissional da saúde, possibilitando-o modificar e redirecionar sua abordagem para esse grupo singular, oferecendo apoio sensível e especializado.

\section{CONSIDERAÇÕES FINAIS}

A realização de uma revisão integrativa sobre práticas alimentares de filhos de mães adolescentes possibilitou o levantamento e análise de publicações no contexto mundial. Além do Brasil, nenhuma publicação latina foi identificada, revelando uma carência de estudos nestes países. A escassez de artigos demonstra que a pesquisa sobre este tema ainda não está consolidada, sobretudo no que diz respeito à alimentação complementar, desvelando uma lacuna na produção mundial sobre o tema.

Novas pesquisas, portanto, precisam ser desenvolvidas com foco em mães adolescentes, devendo-se explorar a alimentação infantil além das avaliações de consumo. A realização de estudos em diferentes etnias possibilitaria o conhecimento da diversidade cultural, auxiliando o planejamento de ações específicas para cada grupo social.

\section{REFERÊNCIAS}

1. Leão E, Corrêa EJ, Mota JAC, Viana MB. Pediatria ambulatorial. $4^{\text {a }}$ ed. Belo Horizonte: Coopmed; 2005.

2. Brasil. Ministério da Saúde. Secretaria de Atenção à Saúde. Marco legal: saúde, um direito de adolescentes. Cadernos Técnicos, série A, Normas e Manuais Técnicos. Brasília: Ministério da Saúde; 2007.

3. Recife. Prefeitura do Recife. Perfil epidemiológico da criança e do adolescente. Recife: Secretaria de Saúde, Diretoria de Vigilância à Saúde; 2009.

4. Vieira MLF, Pinto e Silva JLC, Barros Filho AA. A amamentação e a alimentação complementar de filhos de mães adolescentes são diferentes das de mães adultas? J. Pediatr. (Rio J.) 2003;79(4):317-24.

5. Bergamaschi SFF, Praça NS. Vivência da puérpera adolescente no cuidado do recém-nascido, no domicílio. Rev Esc Enferm USP 2008;42(3): 454-60.

6. Bercini LO, Masukawa MLT, Martins MR, Labegalini MPC, Alves NB. Alimentação da criança no primeiro ano de vida, em Maringá, PR. Cienc. Cuid. Saúde 2007;6(2): 404-10.

7. World Health Organization. Infant and young child feeding: model chapter for textbooks for medical students and allied health professionals. Geneva: WHO; 2009.

8. Feldman-Winter L, Shaikh U. Optimizing breastfeeding promotion and support in adolescent mothers. J. Hum. Lact. 2007;23(4):362-7.

9. World Health Organization. Adolescent pregnancy [Internet]. Geneva: WHO; 2008 [cited 2010 Apr. 28]. Available from: http://www.who.int/making_pregnancy_safer/ events/2008/mdg5/adolescent_preg.pdf

10. Mendes KDS, Silveira RCCP, Galvão. Revisão integrativa: método de pesquisa para a incorporação de evidências na saúde e na enfermagem. Texto \& contexto enferm. 2008;17(4): 758-64.

11. Silveira CS, Zago MMF. Pesquisa brasileira em enfermagem oncológica: uma revisão integrativa. Rev. Lat-Am. Enfermagem 2006;14(4): 614-9.

12. Souza MT, Silva MD, Carvalho R. Revisão integrativa: o que é e como fazer. Einstein (São Paulo) 2010;8(1): 102-6.

13. Hannon PR, Willis SK, Bishop-Townsend V, Martinez IM. African-American and Latina adolescent mothers' infant feeding decisions and breastfeeding practices: a qualitative study. J. Adolesc .Health. 2000;26(6): 399-407.

14. Flick U. Introdução à pesquisa qualitativa. $3^{\mathrm{a}} \mathrm{ed}$. Porto Alegre: Artmed; 2009.

15. Brasil. Ministério da Saúde. Secretaria de Atenção à Saúde. Departamento de Atenção Básica. Saúde da criança: nutrição infantil - aleitamento materno e alimentação complementar. Cadernos de Atenção Básica, série A, Normas e Manuais Técnicos. Brasília: Ministério da Saúde; 2009.

16. Baião MR, Deslandes SF. Alimentação na gestação e puerpério. Rev. Nutr. 2006;19(2): 245-53.

17. Wambach KA, Cohen SM. Breastfeeding experiences of urban adolescent mothers. J. Pediatr. Nurs. 2009;24(4): 244-54.

18. Spear HJ. Breastfeeding behaviors and experiences of. MCN Am J Matern Child Nurs 2006;31(2): 106-13.

19. Dykes F, Moran VH, Burt S, Edwards J. Adolescent mothers and breastfeeding: experiences and support needs an exploratory study. J. Hum. Lact. 2003;19(4): 391-400.

20. Nelson AM. Adolescent attitudes, beliefs and concerns regarding breastfeeding. MCN Am J Matern Child Nurs 2009;34(4): 249-55. 
21. Black MM, Siegel EH, Abel Y, Bentley ME. Home and videotape intervention delays early complementary feeding among adolescent mothers. Pediatrics 2001;107(5):1-8.

22. Mossman M, Heaman M, Dennis C, Morris M. The influence of adolescent mothers' breastfeeding confidence and attitudes on breastfeeding initiation and duration. J. Hum. Lact. 2008;24(3): 267-77.

23. Marques RFSV, Cunha ICC, Aragón MG, Peixoto VS. Fatores relacionados às dificuldades no aleitamento materno entre mães adolescentes da Fundação Santa Casa de Misericórdia do Pará. Rev. Para. Med. 2008;22(1): 57-62.

24. Sepka GC, Gasparelo L, Fernandes e Silva AB, Mascarenhas TT. Promoção do aleitamento materno com mães adolescentes: acompanhando e avaliando essa prática. Cogitare Enferm. 2007;12(3): 313-22.

25. Lima AP, Javorski M. Amamentação interrompida: vivência de mulheres-mães. Ver. Enferm. UFPE on line [periódico na Internet]. 2010 jan/mar [citado em 21 maio 2010];4(1): 227-35. Disponível em: http://www.ufpe.br/revistaenfermagem/index.php/revista/article/viewFile/729/464
26. Susin LRO, Giugliane ERJ, Kummer SC. Influência das avós na prática do aleitamento materno. Rev. Saúde Pública 2005;39(2): 141-7.

27. Frota DAL, Marcopito LF. Amamentação entre mães adolescentes e não adolescentes, Monte Carlos, MG. Rev. Saúde Pública 2004;38(1): 85-92.

28. Bernardi JLD, Jordão RE, Barros Filho AA. Alimentação complementar de lactentes em uma cidade desenvolvida no contexto de um país em desenvolvimento. Rev. Panam. Salud Pública 2009;26(5): 405-11.

29. Fuzeto KLR, Oliveira ACL. Comparação da prática do aleitamento materno e da alimentação complementar entre mães adolescentes e adultas, Curitiba/PR. Cadernos da Escola de Saúde [periódico na Internet]. 2010 [citado em 09 jul 2010];3: 1-16. Disponível em: http:// apps.unibrasil.com.br/revista/index.php/saude/article/ viewFile/334/264

30. Lemos MCM, Dallacosta MC. Hábitos alimentares de adolescentes: conceitos e práticas. Arq. Ciênc. Saúde Unipar 2005;9(1): 3-9. 


\section{ERRATAS}

Vol.64 № 4 jul./ago. 2011

\begin{tabular}{|c|c|c|}
\hline PÁGINA & ONDE SE LÊ & LEIA-SE \\
\hline 751 & Talita Aquira Santos Lima & Talita Aquira dos Santos Lima \\
\hline 751 & $\begin{array}{l}\text { Conclui-se que a produção científica sobre o idoso } \\
\text { longevo ainda é singela e irrelevante. }\end{array}$ & $\begin{array}{l}\text { Conclui-se que a produção científica sobre o idoso } \\
\text { longevo ainda é singela, mas relevante. }\end{array}$ \\
\hline 751 & $\begin{array}{l}\text { One concluded that the scientific production on } \\
\text { long-lived elderly person is still small and irrelevant. }\end{array}$ & $\begin{array}{l}\text { One concluded that the scientific production on } \\
\text { long-lived elderly person is still small, but relevant. }\end{array}$ \\
\hline 751 & $\begin{array}{l}\text { Se concluye que la producción científica sobre el } \\
\text { mayor de } 80 \text { anos es todavía aun simple e irrelevante. }\end{array}$ & $\begin{array}{l}\text { Se concluye que la producción científica sobre el } \\
\text { mayor de } 80 \text { anos es todavía aun simple, más relevante. }\end{array}$ \\
\hline 751 & $\begin{array}{l}\text { AUTOR CORRESPONDENTE } \\
\text { Talita Aquira Santos Lima talyaquira@gmail.com }\end{array}$ & $\begin{array}{l}\text { AUTOR CORRESPONDENTE } \\
\text { Talita Aquira dos Santos Lima talyaquira@hotmail.com }\end{array}$ \\
\hline \multicolumn{3}{|c|}{ Vol.64 № 5 set./out. 2011} \\
\hline PÁGINA & ONDE SE LÊ & LEIA-SE \\
\hline 912 & $\begin{array}{l}\text { Extraído de Pesquisa de Iniciação Científica, financiada } \\
\text { pela FAPEMIG, intitulada "Dimensionamento de pessoal de } \\
\text { enfermagem frente à classificação dos clientes hospitalizados } \\
\text { em um hospital de ensino". }\end{array}$ & $\begin{array}{l}\text { Extraído da Dissertação "Práticas alimentares no primeiro } \\
\text { ano de vida na percepção de mães adolescentes", Programa } \\
\text { de Pós-Graduação em Saúde da Criança e do Adolescente, } \\
\text { Universidade Federal de Pernambuco, } 2011 .\end{array}$ \\
\hline
\end{tabular}

